\title{
Treatment Outcomes of Proliferative vs. Non- proliferative Adult Lupus Nephritis: A 10-Year Follow-Up
} Mohamed Zahab ${ }^{1}$, Mohammed A. Fouda $^{1}$, Yasser Elhendy ${ }^{2}$, Amir Elokely ${ }^{2}$, Mona Abdul Rahim ${ }^{3}$, Ayman
F. Refaie ${ }^{1}$, Sami Alobaidi $^{4}$, Ahmed Akl $^{1}$

1. Nephrology Department, Urology \& Nephrology Center, Mansoura, EGY 2. Internal Medicine Department, Zagazig University, Zagazig, EGY 3. Pathology Department, Urology \& Nephrology Center, Mansoura, EGY 4. Department of Medicine, College of Medicine, University of Jeddah, Jeddah, SAU

Corresponding author: Ahmed Akl, aiak12001@yahoo.com

\section{Abstract \\ Introduction}

Systemic lupus erythematosus (SLE) is a systemic disease with clinically heterogeneous outcomes. Lupus nephritis (LN) is a common complication of SLE. LN impacts clinical SLE outcomes both directly, in the form of target organ damage, and indirectly, through the adverse effects of immunosuppressive therapy.

\section{Patients \& methods}

The study included 402 SLE cases with biopsy-proven lupus nephritis who were under follow-up for the past 13 years at Mansoura Urology and Nephrology Center, Egypt. We studied the differences in outcome among various LN classes and between 275 proliferative cases and 102 non-proliferative cases.

\section{Results}

Class IV was the main LN class in our series with renal survival of $60 \%$ at 10 years. The major induction regimen after the first biopsy was cyclophosphamide. Mycophenolate mofetil was the main induction and adjunctive regimen after the second biopsy. The mean follow-up period was $6.7+5.2$ years. Higher serum creatinine, proteinuria, activity, and chronicity indices were noted in proliferative LN. Patients suffering from proliferative lesions received higher immunosuppression and demonstrated higher morbidity than those with non-proliferative lesions. Remission was higher among the non-proliferative compared to the proliferative group.

\section{Conclusions}

Serum creatinine, proteinuria, and LN class were the most relevant prognostic factors for renal survival among Egyptian LN patients.

Review began 07/11/2021 Review ended 07/24/2021 Published 08/06/2021

\section{() Copyright 2021}

Zahab et al. This is an open access article distributed under the terms of the Creative Commons Attribution License CC-BY 4.0., which permits unrestricted use, distribution, and reproduction in any medium, provided the original author and source are credited.
Categories: Pathology, Nephrology, Rheumatology

Keywords: systemic lupus, lupus nephritis, outcome in lupus, cyclophosphamide, mycophenolate mofetil, rituximab

\section{Introduction}

Studying systemic lupus erythematosus (SLE) in humans is challenging, as the disease affects several organ systems generating clinically diverse outcomes. The characteristic indication of SLE is a systemic autoimmune response. This is ascribed to abnormal T-cell signaling pathways, reduced thresholds for initiating immune cell responses, and malfunctioning tolerance mechanisms [1]. As regards gender, SLE is often called a "woman's disease" secondary to the striking differences in sex-related prevalence [2]. Lupus nephritis (LN) is not an uncommon consequence of SLE and occurs in 45\%-86\% of SLE patients in Japan and $31 \%-65 \%$ of SLE patients in the United States and European countries [3].

The clinical outcome is influenced by LN both directly and indirectly. The former results in organ damage, whilst the latter arises immunosuppressive therapy having deleterious effects [4]. Based upon their elevated creatinine levels and activity renal index values, renal impairment is more severe in male patients with LN than in female patients with SLE [5].

In LN, the major immunological features consist of a loss of self-tolerance to autoantigens [6]. In addition, the anti-double-stranded DNA antibodies directed against nucleosomes contribute to the glomerular injury [7]. The International Society of Nephrology/Renal Pathology Society (ISN/RPS) histopathological classification of LN continues to guide therapy, and all classes of LN have recently undergone a shift in management [8-9]. After the kidney becomes involved in SLE, the prognoses for lupus patients decline. This 


\section{Materials And Methods}

\section{Patients and methods}

The patients' cohort included 402 cases with biopsy-proven lupus nephritis. In this retrospective evaluation, we selected patients fulfilling American Rheumatology Association (ARA) criteria for the diagnosis of SLE [11] and were under follow-up at Mansoura Urology and Nephrology Center, Mansoura University, for the past 13 years. The LN diagnosis was based on evidence of LN, including (1) Significant proteinuria (proteinuria defined as $>0.5 \mathrm{~g} / \mathrm{day}$ ) and/or (2) Abnormal high serum creatinine associated with urine cellular or granular casts. Renal biopsies were performed when clinical and laboratory signs of renal involvement were observed. The renal biopsies analysis included light microscopy and immunofluorescence. Histological assessments were based on ISN/RPS (2003) [12]. In addition, we studied the differences in outcome between males (n. 40) and females (n. 363) in addition to the differences in outcome between proliferative (n. 275) and non-proliferative (n. 102).

Complete response was considered once serum creatinine return to baseline values and a decline in the urine protein/creatinine ratio (uPCR) to $<500 \mathrm{mg} / \mathrm{g}$ ( $<50 \mathrm{mg} / \mathrm{mmol}$ ) [13]. Partial response is defined as stabilization $( \pm 25 \%)$ or improvement of creatinine, but not a return to normal, in addition to a $\geqslant 50 \%$ decrease in uPCR. If nephrotic-range proteinuria ( $\mathrm{uPCR} \geqslant 3000 \mathrm{mg} / \mathrm{g}[\geqslant 300 \mathrm{mg} / \mathrm{mmol}]$ ) was observed, improvement required a $\geqslant 50 \%$ reduction in uPCR and a $\mathrm{UPCR}<3000 \mathrm{mg} / \mathrm{g}$ ( $<300 \mathrm{mg} / \mathrm{mmol}$ ) [13]. A sustained $25 \%$ increase in creatinine is widely used to define treatment failure but has not been validated [13].

The treatment of LN consisted of different combinations of oral steroids, azathioprine, and intravenous cyclophosphamide (The Euro-Lupus regimen). Furthermore, some patients received mycophenolate mofetil (MMF), plasmapheresis, intravenous methyl-prednisolone, cyclosporine A, and rituximab, according to clinical indications. The majority of patients in the non-proliferative group received cyclosporin.

\section{Statistical analysis}

The data extracted from the study were analyzed using SPSS for Windows (release 16 SPSS Inc., Chicago, III). Qualitative data were formulated in cross-tabulation, and quantitative data were presented in terms of arithmetic mean and standard deviation. Bivariate techniques were used for the initial evaluation of contrast. Thus, the chi-square and Fisher's exact test were used to compare qualitative variable frequencies, and the unpaired T-test was used to compare the means of two quantitative variables. Multivariate analysis was done using a Cox regression analysis. A p-value $<0.05$ was considered significant.

\section{Results}

The mean age of SLE onset was $24.50 \pm 9$.9 years while the mean age of LN onset was $26.57 \pm 8.8$ years. The time between SLE and LN was $24.47 \pm 68.6$ months. Male patients constituted $10.2 \%$ of the total cases. Most of our patients were virology negative (93.5\%) regarding hepatitis C antibody, hepatitis B surface antigen, and human immunodeficiency virus antibodies. The family history of SLE was positive in $9.5 \%$ of our cohort. Mean basal serum creatinine was $1.37 \pm 1.5 \mathrm{mg} / \mathrm{dl}$, and proteinuria (mean+SD) was $4 \pm 2.7 \mathrm{gm} / \mathrm{day}$. About $4 \%$ of our patients did not proceed for renal biopsy. The most common cause of avoidance of renal biopsy was antiphospholipid antibody syndrome (APLS) on warfarin (1\%) and proteinuria <0.5 gm (Table 1). 


\section{Cureus}

\begin{tabular}{|c|c|}
\hline & Lupus nephritis cases $($ No. $=402)$ \\
\hline 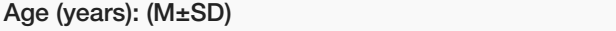 & $33.24 \pm 9.8$ \\
\hline \multicolumn{2}{|l|}{ Sex: No. (\%) } \\
\hline Male & $41(10.2 \%)$ \\
\hline Female & $361(89.8 \%)$ \\
\hline Body mass index (M $\pm S D)$ & $32.92 \pm 21.8$ \\
\hline \multicolumn{2}{|l|}{ Locality: No. (\%) } \\
\hline Dakahlia & $231(57.5 \%)$ \\
\hline Damietta & $61(15.2 \%)$ \\
\hline Kafe-Elskeikh & $42(10.4 \%)$ \\
\hline Gharbia & $33(8.2 \%)$ \\
\hline Others & $35(8.7 \%)$ \\
\hline Age at onset of SLE (years): (M $\pm S D)$ & $24.50 \pm 9.9$ \\
\hline Age at onset of LN (years): (M $\pm S D)$ & $26.57 \pm 8.8$ \\
\hline Time between onset of SLE and LN (month): (M $\pm S D$ ) & $24.47 \pm 68.6$ \\
\hline \multicolumn{2}{|l|}{ Virology: No (\%) } \\
\hline Negative & 376 (93.5\%) \\
\hline Positive & $22(5.5 \%)$ \\
\hline Missing: & $4(1 \%)$ \\
\hline \multicolumn{2}{|l|}{ Family history of SLE: No (\%) } \\
\hline YES & $38(9.5 \%)$ \\
\hline NO & $363(90.3 \%)$ \\
\hline Missing & $1(0.2 \%)$ \\
\hline
\end{tabular}

TABLE 1: Demographic characteristics of lupus nephritis patients

Sixty-seven percent of our patients had proliferative LN. Class IV was the main LN class (43.2\%) in our patient series. Class IV renal survival was $65 \%$ at 10 years, and class VI demonstrated the worst outcome (Figures 1-2). 


\section{Cureus}

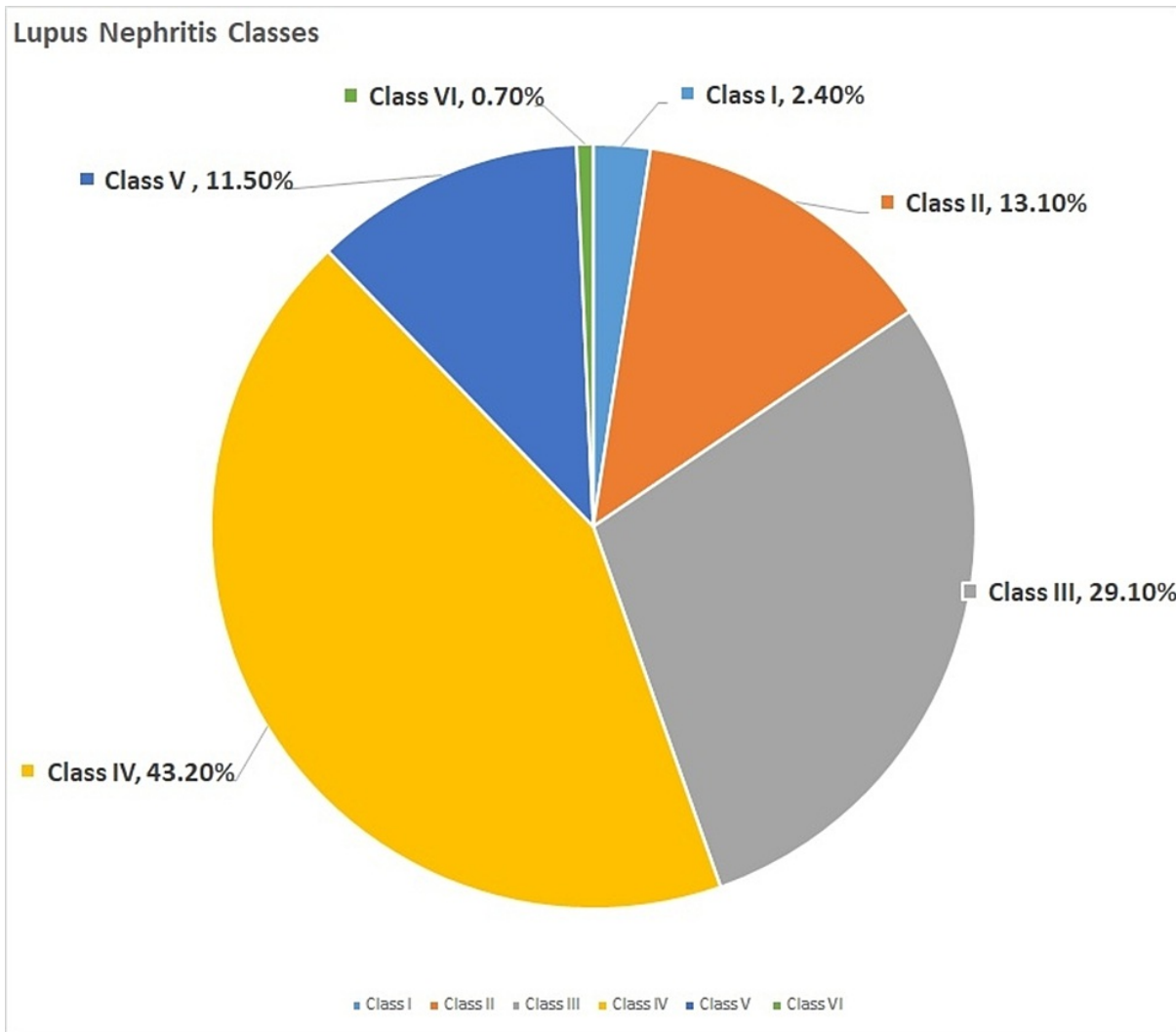

FIGURE 1: Prevalence of different LN classes among adult patients This chart demonstrates the prevalence of different histopathological classes among lupus nephritis (LN) patients. 


\section{Cureus}

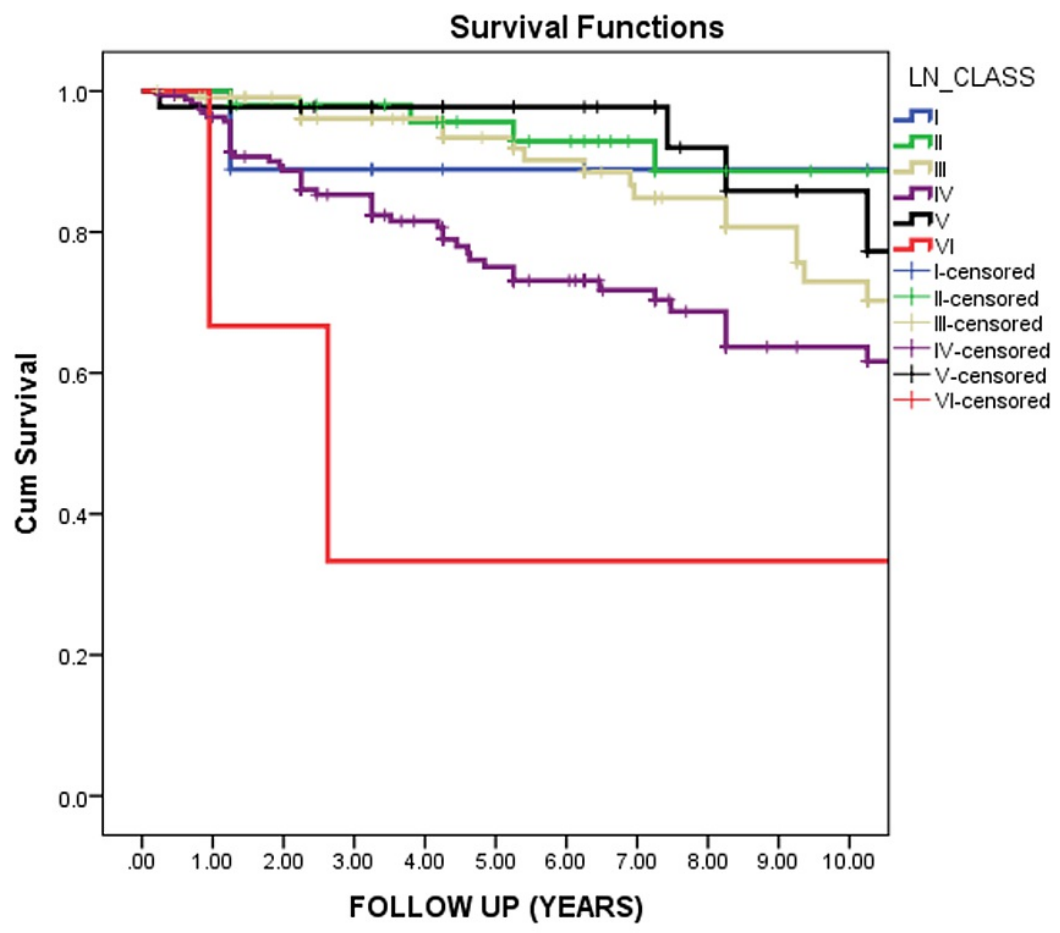

\section{FIGURE 2: Survival of different LN classes among adult patients}

This curve demonstrated the significant difference in renal survival between different lupus nephritis (LN) classes. The best survival for classes 1,2,5 and the worst for classes 3,4,6.

$(P$-value $=0.000)$

About 22\% of our patients received pulse steroids, and the rest received a daily dose of prednisolone $1 \mathrm{mg} / \mathrm{kg}$ of body weight (maximum dose $80 \mathrm{mg} /$ day). Regarding induction therapy, $46 \%$ of patients received cyclophosphamide, and 19\% received MMF. The primary induction regimen after the first biopsy was cyclophosphamide. Mycophenolate mofetil was the primary induction and adjunctive regimen after the second biopsy among LN patients.

Regarding maintenance therapy, $55.6 \%$ of our patients received steroid + azathioprine while $25.8 \%$ received steroid + MMF. Seventy-two percent of our cases received hydroxychloroquine. The mean follow-up period was about $6.7+5.2$ years.

At first presentation, serum creatinine $>1.4 \mathrm{mg} / \mathrm{dl}$ was associated with highly significant worse renal survival $(\mathrm{P}<0.001)$, and nephrotic range proteinuria was associated with the worst renal outcome $(\mathrm{p}=0.006)$ (Figures $3-4)$. 


\section{Cureus}

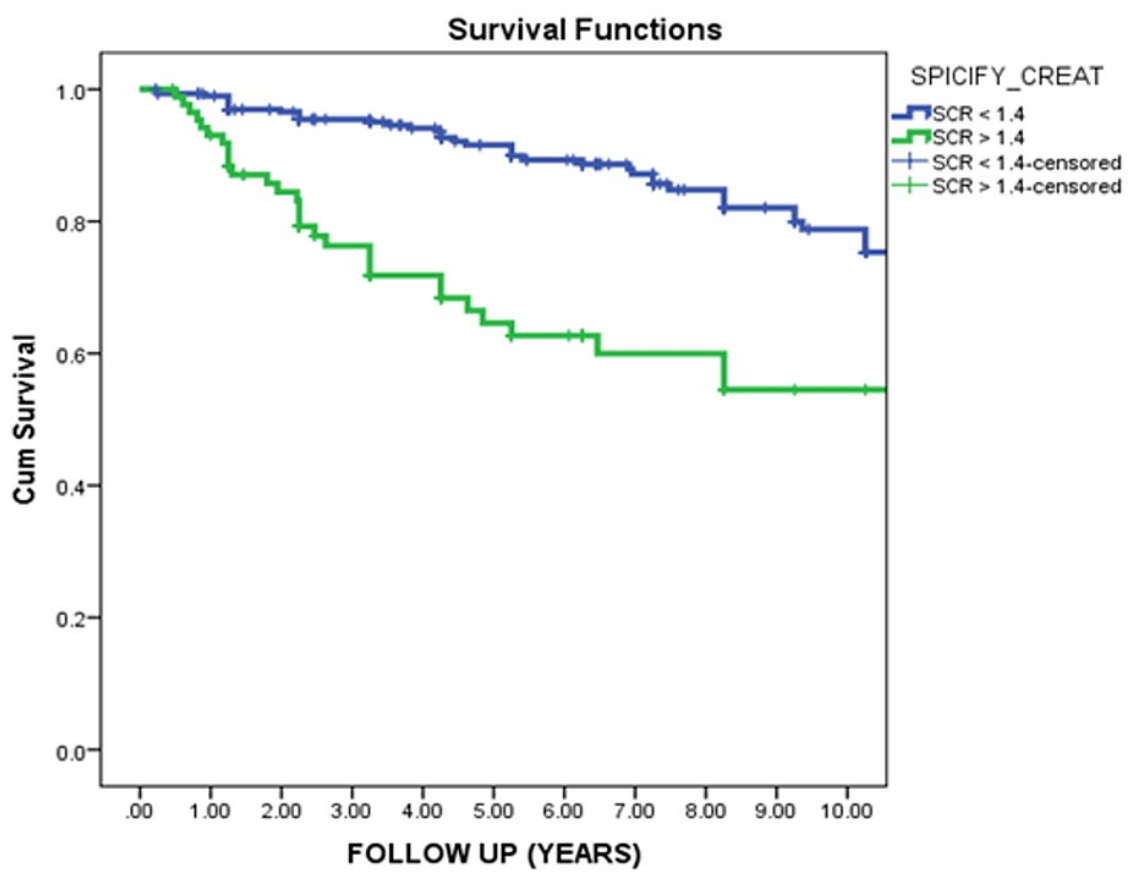

FIGURE 3: Renal survival in relation to the level of serum creatinine at first presentation

This curve demonstrated that serum creatinine at the onset of the disease typically affects renal survival. Patients with normal serum creatinine $(<1.4 \mathrm{mg} / \mathrm{dl})$ showed significantly better renal survival than patients with high serum creatinine $(>1.4 \mathrm{mg} / \mathrm{dl})$ ( $p$-value 0.000$)$. 


\section{Cureus}

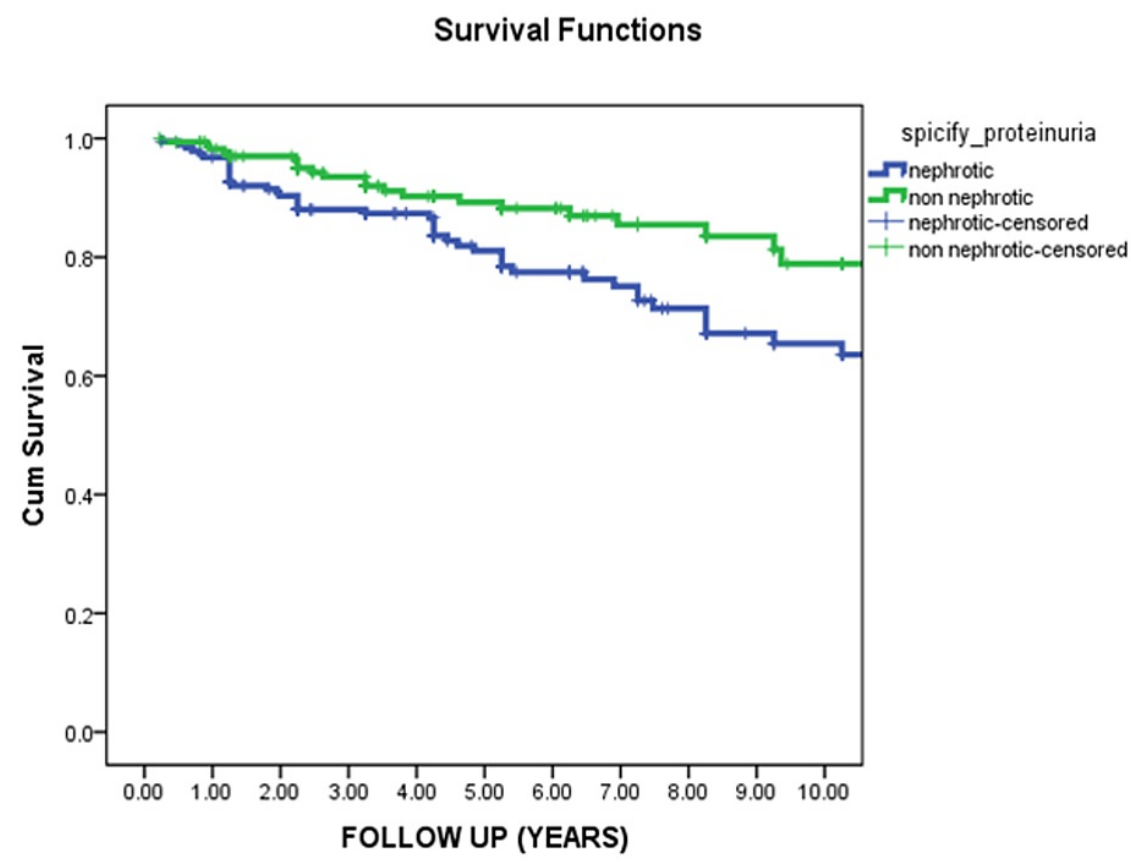

FIGURE 4: Renal survival in relation to the level of proteinuria at first presentation

This curve demonstrated that the level of proteinuria at the onset of the disease typically affects renal survival. Patients with non-nephrotic range proteinuria showed significantly better renal survival than patients with nephrotic range proteinuria ( $p$-value 0.006$)$.

Hypertension and infection were the most common complications among our patients. APLS was seen in about $17 \%$ of our cases. Most of the in-remission cases were class I; also, most cases in relapse, CKD, and/or reaching hemodialysis were class IV cases. The highest percentage of partial remission was among class $\mathrm{V}$ and constituted $38 \%$ of our cases.

Table 2 shows the demographic characteristics of proliferative and non-proliferative lupus patients. Demographic characteristics were comparable in both groups. Proliferative LN was associated with statistically significant higher basal serum creatinine, basal proteinuria, and activity and chronicity indices. 


\section{Cureus}

\begin{tabular}{|c|c|c|c|}
\hline & $\begin{array}{l}\text { Proliferative lupus nephritis } \\
\text { (No.=276) }\end{array}$ & $\begin{array}{l}\text { Non-proliferative lupus nephritis } \\
\text { (No.=103) }\end{array}$ & $\begin{array}{l}P \text { - } \\
\text { value }\end{array}$ \\
\hline Age (years): (M $\pm S D)$ & $33 \pm 9.8$ & $33.38 \pm 9.2$ & 0.7 \\
\hline Body mass index: (M $\pm S D)$ & $29.30 \pm 6.2$ & $30.33 \pm 6.6$ & 0.1 \\
\hline \multicolumn{4}{|l|}{ Locality: No. (\%) } \\
\hline Dakahlia & $155(56.1 \%)$ & $60(58.3 \%)$ & \multirow{5}{*}{0.74} \\
\hline Damietta & $42(15.2 \%)$ & $15(14.6 \%)$ & \\
\hline Kafe-Elskeikh & $28(10.1 \%)$ & $13(12.6 \%)$ & \\
\hline Gharbia & $21(7.6 \%)$ & $10(9.7 \%)$ & \\
\hline Others & $30(10.8 \%)$ & $5(4.8 \%)$ & \\
\hline Age of onset of SLE (years): (M $\pm S D)$ & $24.81 \pm 10.54$ & $24.12 \pm 7.85$ & 0.26 \\
\hline Age of onset of LN (years): (M $\pm S D$ ) & $26.65 \pm 8.78$ & $26.31 \pm 8.22$ & 0.21 \\
\hline $\begin{array}{l}\text { Time between onset of SLE and L.N (month): } \\
(M \pm S D)\end{array}$ & $21.63 \pm 75.53$ & $26.31 \pm 46.44$ & 0.4 \\
\hline \multicolumn{4}{|l|}{ Virology: No. (\%) } \\
\hline Negative & $261(94.56 \%)$ & $96(93.20 \%)$ & \multirow{2}{*}{0.39} \\
\hline Positive & $15(5.43 \%)$ & $7(6.79 \%)$ & \\
\hline Positive Family history of SLE: No.(\%) & $25(9.1 \%)$ & $11(10.7 \%)$ & 0.38 \\
\hline $\begin{array}{l}\text { Positive Family history of renal disease: No. } \\
\text { (\%) }\end{array}$ & $34(12.3 \%)$ & $10(9.7 \%)$ & 0.30 \\
\hline \multicolumn{4}{|l|}{ Complications: No. (\%) } \\
\hline Hypertension & $221(80.1 \%)$ & $63(61.2 \%)$ & 0.001 \\
\hline Diabetes mellitus & $25(9.1 \%)$ & $10(9.7 \%)$ & 0.4 \\
\hline Thrombotic events & $36(13 \%)$ & $5(4.9 \%)$ & 0.01 \\
\hline Anti-phospholipid syndrome & $50(18.1 \%)$ & $10(9.7 \%)$ & 0.03 \\
\hline $\begin{array}{l}\text { Neurological (stroke, sinus thrombosis, } \\
\text { cerebritis) }\end{array}$ & $31(11.2 \%)$ & $4(3.9 \%)$ & 0.01 \\
\hline Infection: & $63(22.8 \%)$ & $14(13.6 \%)$ & 0.03 \\
\hline Pneumonia & $41(66.1 \%)$ & $8(57.1 \%)$ & 0.1 \\
\hline Cellulitis & $9(14.2 \%)$ & $2(14.2 \%)$ & 1.0 \\
\hline Intra-abdominal infection & $8(12.7 \%)$ & $1(0.07 \%)$ & 0.08 \\
\hline Herpes zoster & $4(6.3 \%)$ & $3(21.4 \%)$ & 0.07 \\
\hline Skin wart & $1(1.6 \%)$ & - & - \\
\hline Malignancy & $(1.8 \%)$ & 2 (1.9\%) & 0.6 \\
\hline
\end{tabular}

TABLE 2: Demographic characteristics of proliferative and non-proliferative lupus nephritis patients

Proliferative LN was significantly associated with worst renal survival than non-proliferative LN ( $p=0.001)$ (Figure 5). 


\section{Cureus}

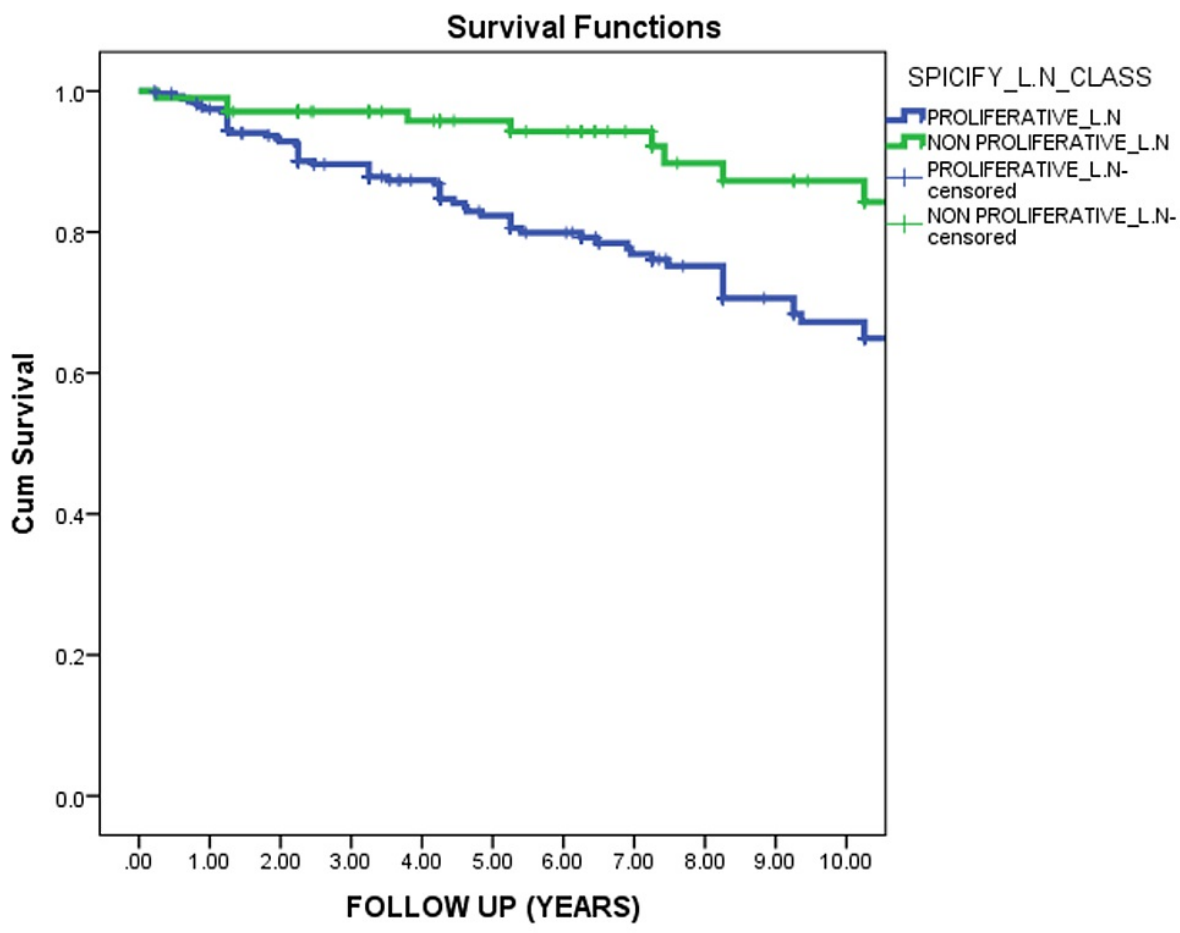

FIGURE 5: Renal survival of proliferative and non-proliferative lupus nephritis

This curve demonstrated that the non-proliferative lupus nephritis group was significantly better in renal survival than the proliferative group $(p-v a l u e=0.001)$.

Cyclophosphamide induction therapy compared to mycophenolate mofetil was associated with significantly better renal survival (Figure 6). 


\section{Cureus}

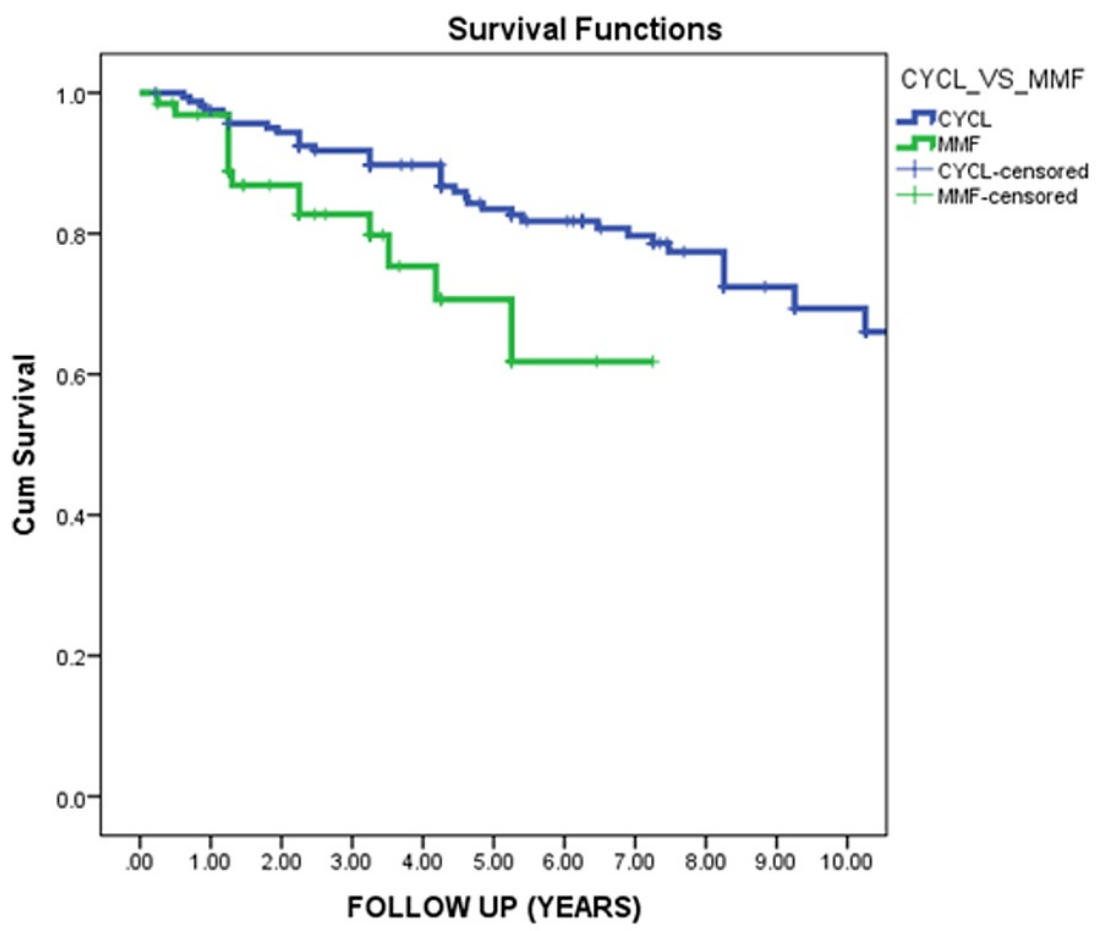

\section{FIGURE 6: Renal survival in relation to the type of induction in proliferative lupus nephritis}

This curve demonstrated that proliferative lupus nephritis who received cyclophosphamide as induction therapy showed significantly better renal survival than patients who received MMF as induction therapy ( $p$ value $=0.006$ ).

MMF: mycophenolate mofetil

Forty-four percent (44\%) of the proliferative and 33\% of non-proliferative cases underwent second biopsy (p-value 0.3). Classes III and IV presented in proliferative LN lesions after the second biopsy to a much greater extent than in the non-proliferative lesions. Significant differences between both groups regarding the use of pulse steroids, induction, and maintenance therapies were noted. Hypertension [221 (80.1\%) vs 63 (61.2\%); $\mathrm{p}<0.001$ ], APLS, infections, and thrombotic and neurological events were observed among the proliferative group (Table 2). CKD and end-stage renal disease (ESRD) were significantly higher among the proliferative group. Serum creatinine was higher at the last follow-up among the proliferative group over the non-proliferative group.

\section{Discussion}

LN is an immune complex type of glomerulonephritis; patients with SLE often experience severe LN [14]. The inflammatory response caused by the accumulation of glomerular immune complexes initially damages the glomeruli, but with time, the damage extends to the renal interstitium. The prognoses for patients with LN deteriorate once the SLE affects the kidneys. Part of this deterioration is attributed to the onset of CKD or ESRD; there is also a heightened risk of CKD leading to cardiovascular disease.

In our study, renal survival among all LN patients was $95 \%$ after the first year, $86 \%$ after five years, $73 \%$ after 10 years, and $60 \%$ after 15 years. This was lower than reported by Al Arfaj et al., who reported high renal survival [15]. In a study from India, Dhir et al. reported that renal survival for Asian Indian patients was $79 \%$, $70 \%$, and $66 \%$ for five, 10, and 15 years, respectively [16]. Another study by Faurschou et al. found that five-, 10 -, and 20 -year renal survivals were $87 \%, 83 \%$, and $73 \%$, respectively [17]. Mok et al. found higher renal survival rates in patients with diffuse proliferative LN received cyclophosphamide [18]. Hypertension was the most common comorbidity found in lupus nephritis patients [19-20].

Proliferative LN was higher among male than female patients (79.5\% versus $72.3 \%$ ); however, the difference was not statistically significant $(\mathrm{p}=0.2)$. In addition, the male gender showed higher activity index in renal biopsies ( $p=0.01$ ). De Carvalho et al. reported that LN appears to cause greater renal damage as revealed by higher serum creatinine and activity renal index values in comparison with SLE in women [5]. Mahmoud et 
al. (2015) reported that the male gender is associated with poor outcomes [21]. In our study, male patients performed poorly regarding activity scores and kidney function but there were no differences in histologybased scores or renal replacement therapy requirements. Wang et al. reported similar data in Chinese males as compared to other nationalities [22].

In our data, proliferative LN showed significantly poorer renal outcomes than in non-proliferative LN patients $(\mathrm{P}=0.001)$. This goes hand in hand with $\mathrm{Al}$ Arfaj et al., who reported that proliferative $\mathrm{LN}$ is associated with poorer outcomes [14]. Also, Pinto et al. reported the aggressive behavior of proliferative nephritis [23]. Regarding induction therapy for proliferative LN patients, we found that induction with cyclophosphamide produced better renal survival than induction with MMF ( $\mathrm{P}=0.006)$. In contrast, El-Shafey et al.'s data suggest MMF as an alternative treatment to induction by pulse cyclophosphamide [24]. However, Onishi et al. deferred the MMF superiority to pulse cyclophosphamide induction [25]. Kallenberg demonstrated that both the Euro-Lupus protocol and MMF with corticosteroids might be considered for remission induction in patients with proliferative LN [26]. Our data suggest that initial serum creatinine level at presentation carries significant predictive value to renal survival. High serum creatinine was found to be associated with poor renal outcomes $[14,26]$.

\section{Conclusions}

In conclusion, our study has the limitations of being a single-center study with a retrospective approach, hence prospective multicentre studies are needed to enforce evidence. Serum creatinine, proteinuria, and LN class are the most relevant prognostic factors for renal survival among LN patients. Management should be tailored according to proliferative or non-proliferative lupus nephritis to maintain efficacy and avoid side effects.

\section{Additional Information \\ Disclosures}

Human subjects: Consent was obtained or waived by all participants in this study. Institutional review board (IRB) of Zagazig University issued approval 154/IRB/2020. A written informed consent was not required, and approved by the institutional review board (IRB) of Zagazig University (154/IRB/2020). No consent was obtained from the participants of the study due to its retrospective design. Animal subjects: All authors have confirmed that this study did not involve animal subjects or tissue. Conflicts of interest: In compliance with the ICMJE uniform disclosure form, all authors declare the following: Payment/services info: All authors have declared that no financial support was received from any organization for the submitted work. Financial relationships: All authors have declared that they have no financial relationships at present or within the previous three years with any organizations that might have an interest in the submitted work. Other relationships: All authors have declared that there are no other relationships or activities that could appear to have influenced the submitted work.

\section{Acknowledgements}

We would like to thank our colleagues and nurses in the Urology and Nephrology Center, Mansoura University, Egypt, for their dedication to the care of patients and critical assessment and management.

\section{References}

1. Grimaldi CM, Hicks R, Diamond B: B cell selection and susceptibility to autoimmunity. J Immunol. 2005, 174:1775-81. 10.4049/jimmunol.174.4.1775

2. Lu LJ, Wallace DJ, Ishimori ML, Scofield RH, Weisman MH: Review: male systemic lupus erythematosus: a review of sex disparities in this disease. Lupus. 2010, 19:119-29. 10.1177/0961203309350755

3. Yokoyama H, Okuyama H, Yamaya H: Clinicopathological insights into lupus glomerulonephritis in Japanese and Asians. Clin Exp Nephrol. 2011, 15:321-30. 10.1007/s10157-011-0434-0

4. Ortega LM, Schultz DR, Lenz O, Pardo V, Contreras GN: Review: lupus nephritis: pathologic features, epidemiology and a guide to therapeutic decisions. Lupus. 2010, 19:557-74. 10.1177/0961203309358187

5. de Carvalho JF, do Nascimento AP, Testagrossa LA, Barros RT, Bonfá E: Male gender results in more severe lupus nephritis. Rheumatol Int. 2010, 30:1311-5. 10.1007/s00296-009-1151-9

6. Bagavant H, Fu SM: Pathogenesis of kidney disease in systemic lupus erythematosus . Curr Opin Rheumatol. 2009, 21:489-94. 10.1097/BOR.0b013e32832efff1

7. O'Flynn J, Flierman R, van der Pol P, et al.: Nucleosomes and C1q bound to glomerular endothelial cells serve as targets for autoantibodies and determine complement activation. Mol Immunol. 2011, 49:75-83. 10.1016/j.molimm.2011.07.020

8. Dooley MA, Falk RJ: Human clinical trials in lupus nephritis. Semin Nephrol. 2007, 27:115-27. 10.1016/j.semnephrol.2006.09.011

9. Uchida K, Nitta K: Recent advances in the treatment of lupus nephritis . Clin Exp Nephrol. 2012, 16:202-13. 10.1007/s10157-011-0556-4

10. Skamra C, Ramsey-Goldman R: Management of cardiovascular complications in systemic lupus erythematosus. Int J Clin Rheumtol. 2010, 5:75-100.

11. Arnett FC, Edworthy SM, Bloch DA, et al.: The American Rheumatism Association 1987 revised criteria for the classification of rheumatoid arthritis. Arthritis Rheum. 1988, 31:315-24. 10.1002/art.1780310302

12. Weening JJ, D'Agati VD, Schwartz MM, et al.: The classification of glomerulonephritis in systemic lupus 
erythematosus revisited. J Am Soc Nephrol. 2004, 15:241-50. 10.1097/01.asn.0000108969.21691.5d

13. Radhakrishnan J, Cattran DC: The KDIGO practice guideline on glomerulonephritis: reading between the (guide)lines--application to the individual patient. Kidney Int. 2012, 82:840-56. 10.1038/ki.2012.280

14. Grammatikos AP, Tsokos GC: Immunodeficiency and autoimmunity: lessons from systemic lupus erythematosus. Trends Mol Med. 2012, 18:101-8. 10.1016/j.molmed.2011.10.005

15. Al Arfaj AS, Khalil N, Al Saleh S: Lupus nephritis among 624 cases of systemic lupus erythematosus in Riyadh, Saudi Arabia. Rheumatol Int. 2009, 29:1057-67. 10.1007/s00296-009-0905-8

16. Dhir V, Aggarwal A, Lawrence A, Agarwal V, Misra R: Long-term outcome of lupus nephritis in Asian Indians. Arthritis Care Res (Hoboken). 2012, 64:713-20. 10.1002/acr.21597

17. Faurschou M, Dreyer L, Kamper AL, Starklint H, Jacobsen S: Long-term mortality and renal outcome in a cohort of 100 patients with lupus nephritis. Arthritis Care Res (Hoboken). 2010, 62:873-80. 10.1002/acr.20116

18. Mok CC, Ying KY, Ng WL, et al.: Long-term outcome of diffuse proliferative lupus glomerulonephritis treated with cyclophosphamide. Am J Med. 2006, 119:355.e25-33. 10.1016/j.amjmed.2005.08.045

19. Teh CL, Ling GR, Aishah WS: The Sarawak lupus cohort: clinical features and disease patterns of 633 SLE patients in a single tertiary centre from East Malaysia. Rheumatol Int. 2015, 35:153-7. 10.1007/s00296-014$3057-4$

20. Shaharir SS, Mustafar R, Mohd R, Mohd Said MS, Gafor HA: Persistent hypertension in lupus nephritis and the associated risk factors. Clin Rheumatol. 2015, 34:93-7. 10.1007/s10067-014-2802-0

21. Mahmoud GA, Zayed HS, Ghoniem SA: Renal outcomes among Egyptian lupus nephritis patients: a retrospective analysis of 135 cases from a single centre. Lupus. 2015, 24:331-8. 10.1177/0961203314567751

22. Wang YF, Xu YX, Tan Y, Yu F, Zhao MH: Clinicopathological characteristics and outcomes of male lupus nephritis in China. Lupus. 2012, 21:1472-81. 10.1177/0961203312458467

23. Pinto-Peñaranda LF, Duque-Caballero V, Márquez-Hernández JD, Muñoz-Grajales C, Velásquez-Franco CJ: Predictive factors for low rate of remission in a population of Colombian patients with severe proliferative lupus nephritis. Clin Rheumatol. 2015, 34:897-903. 10.1007/s10067-015-2864-7

24. El-Shafey EM, Abdou SH, Shareef MM: Is mycophenolate mofetil superior to pulse intravenous cyclophosphamide for induction therapy of proliferative lupus nephritis in Egyptian patients?. Clin Exp Nephrol. 2010, 14:214-21. 10.1007/s10157-010-0270-7

25. Onishi A, Sugiyama D, Tsuji G, et al.: Mycophenolate mofetil versus intravenous cyclophosphamide for induction treatment of proliferative lupus nephritis in a Japanese population: a retrospective study. Mod Rheumatol. 2013, 23:89-96. 10.1007/s10165-012-0634-9

26. Kallenberg CG: Pro: cyclophosphamide in lupus nephritis. Nephrol Dial Transplant. 2016, 31:1047-52. 10.1093/ndt/gfw069 